\title{
CLePAPS: Fast Pair Alignment of Protein Structures Based on Conformational Letters
}

\author{
Sheng Wang ${ }^{1}$, Wei-Mou Zheng ${ }^{1,2}$ \\ ${ }^{1}$ Institute of Theoretical Physics, Academia Sinica, Beijing 100080, China \\ ${ }^{2}$ To whom correspondence should be addressed
}

E-mail addresses: zheng@itp.ac.cn

\begin{abstract}
Fast, efficient and reliable algorithms for pairwise alignment of protein structures are in ever increasing demand for analyzing the rapidly growing data of protein structures. CLePAPS is a tool developed for this purpose. It distinguishes itself from other existing algorithms by the use of conformational letters, which are discretized states of $3 \mathrm{D}$ segmental structural states. A letter corresponds to a cluster of combinations of the three angles formed by $C_{\alpha}$ pseudobonds of four contiguous residues. A substitution matrix called CLESUM is available to measure similarity between any two such letters. CLePAPS regards an aligned fragment pair (AFP) as an ungapped string pair with a high sum of pairwise CLESUM scores. Using CLESUM scores as the similarity measure, CLePAPS searches for AFPs by simple string comparison. The transformation which best superimposes a highly similar AFP can be used to superimpose the structure pairs under comparison. A highly scored AFP which is consistent with several other AFPs determines an initial alignment. CLePAPS then joins consistent AFPs guided by their similarity scores to extend the alignment by several 'zoom-in' iteration steps. A follow-up refinement produces the final alignment. CLePAPS does not implement dynamic programming. The utility of CLePAPS is tested on various protein structure pairs.
\end{abstract}

Key words: Protein structure; pairwise structure alignment; protein conformational alphabet.

\section{Introduction}

The comparison of protein structures has been an extremely important problem in structural and evolutional biology. The detection of local or global structural similarity between a new protein and a protein with known function allows the prediction of the new protein's function. Since protein structures are better conserved than amino acid sequences, remote homology is detectable more reliably by comparing structures. Structural comparison methods are useful for organizing and classifying known structures, and for discovering structure patterns and their correlation with sequences.

The common goal of all structure alignment methods is to identify a set of residue pairs from each protein that are structurally similar, or to find the optimal correspondence between the atoms in two molecular structures. An exhaustive search for such atomic correspondence between two structures is intractable, and various heuristics have been developed. For example, to lower the dimensionality of the problem, DALI identifies interaction patterns of fragment pairs, $[1,2]$ VAST describes secondary structure elements (SSEs) as a set of vectors, $[3,4]$ while CE designates short aligned fragment pairs (AFPs) of local structural similarities.[5] There are several excellent reviews, e.g. [6] and [7].

For a given correspondence of two point sets, finding the best rigid transposition to superpose the correspondences can be easily done by using a closed-form solution based on singular value decomposition. $[8,9]$ When the transformation between the two sets is given, the problem to find the correspondences (of $\epsilon$-congruence at the maximal or average error tolerance $\epsilon$ ) is rather straightforward. However, when aligning 
two protein structures, at the beginning we know neither the transformation nor the correspondence. A few methods, like DALI and CE, directly search for a good alignment. Many methods start with an initial correspondence (seed matches), from which the optimal transformation for the correspondence is determined. The transformation is then used to update the correspondence. The procedure of progressively building up larger correspondence is iterated until the best correspondence is finally found. The methods vary in the way of seed finding and correspondence updating. There may be an optional follow-up refinement of alignments. A typical example is ProSup.[10]

Protein structural alignment involves the geometric representation of structures. In most cases, only the backbone of pseudobonds formed by $C_{\alpha}$ atoms is considered. Coordinates of $C_{\alpha}$ atoms, which change under translation and rotation in 3D space, are not geometric invariants. Distances used by DALI are the intrinsic property of a geometric object. The bending and torsion angles of pseudobonds, as the chain counterparts of curvature and torsion of a smooth curve, are also geometric invariants. In VAST, SSEs are replaced by the vectors of their axes. This vector representation speeds up the computation, but has low precision for structural elements.

Many tools find AFPs of local similarity as preliminary correspondences. Local similarity is a necessary, but insufficient, condition for global structural alignment. Structurally similar fragments found in a pair of proteins form the basis objects for further examination of their consistency in the spacial arrangement. Consistent pieces then may be joined to obtain a global alignment. Different methods use various criteria and strategies for seed matching, consistency checking and pieces merging. Generally, a stringent criterion for local similarity would create less objects of seed matches, and hence speed up the merging process. However, it would miss some substructures constituting the final global alignment. On the other hand, due to the insufficiencies of local similarity in the global alignment, too loose conditions of local similarity would overload the later filtering task. One has to balance sensitivity with specificity, and make a compromise between efficiency and accuracy.

A way to represent structures is to use conformational alphabets, which are discretized conformational states of certain fragment units of protein backbones.[11]-[16] Our conformational alphabet of 17 letters is obtained by clustering based on the distribution of the two bending angles and one torsion angle formed by three pseudobonds of the quadrupeptide unit. The description by conformational letters provides a good balance between accuracy and simplicity, and converts a 3D structure to a $1 \mathrm{D}$ sequence of letters. Substitution matrices such as the popular PAM and BLOSUM are essential to amino acid sequence alignment algorithms. Without a conformational substitution matrix the use of a conformational alphabet is very limited. In order to implement fast structural comparison in terms of conformational alphabets, we have derived a substitution matrix of conformational letters called CLESUM from a representative pairwise aligned structure set of the FSSP (families of structurally similar proteins) database of Holm and Sander.[17] It has been verified that CLESUM aptly measures the similarity between conformational letter states.[16]

Despite the existence of various pairwise structural alignment algorithms, fast, efficient and reliable algorithms for pairwise alignment are in ever increasing demand for analyzing the rapidly growing data of protein structures. Here we report a tool called CLePAPS developed for fast pairwise alignment of protein structures by fully using our conformational alphabet and its substitution matrix CLESUM. CLePAPS regards an AFP as an ungapped string pair with a high sum of pairwise CLESUM scores. Using CLESUM scores as the similarity measure, CLePAPS searches for AFPs by simple string comparison. Taking highly similar AFPs as a pivot to determine the transformation for superposition, CLePAPS collects consistent AFPs under the guide of their similarity scores to extend the alignment.

\section{Methods}

CLePAPS uses a 3D structure coding of protein backbones consisting of $C_{\alpha}$ pseudobonds. The flow chart of CLePAPS is shown in Fig. 1. 


\subsection{Conformational alphabet}

Three contiguous $C_{\alpha}$ atoms determines two pseudobonds and a banding angle between them. Four contiguous $C_{\alpha}$ atoms, say $a, b, c$ and $d$, determine two such bending angles and a torsion angle which is the dihedral angle between the two planes of triangles $a b c$ and $b c d$. By using a mixture model for the density distribution of the three angles, the local structural states have been clustered as 17 discrete conformational letters of a protein structural alphabet. The centers $(\mu)$, inverse covariance matrices $\left(\Sigma^{-1}\right)$ and weights $(\pi)$ of the clusters for these conformational letters in the phase space spanned by the three angles $\left(\theta, \tau, \theta^{\prime}\right)$, are listed in Table 1.

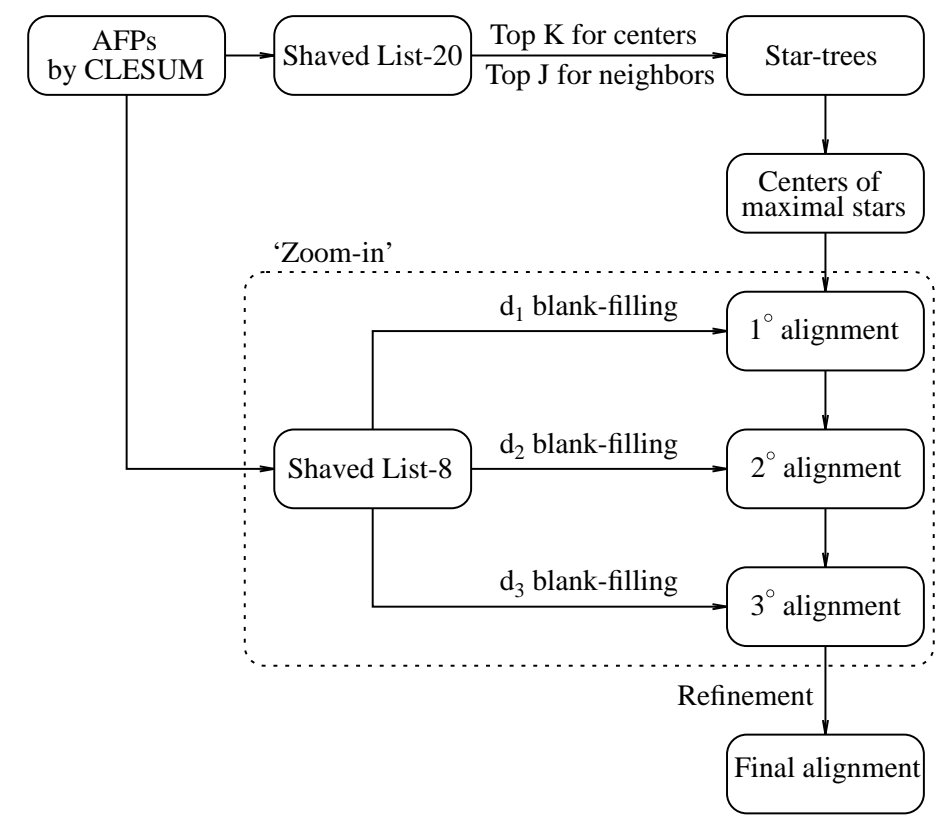

Fig. 1 Flow chart of the CLePAPS algorithm.

Table 1. The 17 conformational states from the mixture model. Angles are in radians.

\begin{tabular}{|c|c|c|c|c|c|c|c|c|c|c|c|}
\hline \multirow[b]{2}{*}{ State } & \multirow[t]{2}{*}{$\pi$} & \multirow[t]{2}{*}{$|\Sigma|^{-1 / 2}$} & \multicolumn{3}{|c|}{$\mu$} & \multicolumn{6}{|c|}{$\boldsymbol{\Sigma}^{-1}$} \\
\hline & & & $\theta$ & $\tau$ & $\theta^{\prime}$ & $\theta \theta$ & $\tau \theta$ & $\tau \tau$ & $\theta^{\prime} \theta$ & $\theta^{\prime} \tau$ & $\theta^{\prime} \theta^{\prime}$ \\
\hline $\mathrm{I}$ & 8.2 & 1881 & 1.52 & 0.83 & 1.52 & 275.4 & -28.3 & 84.3 & 106.9 & -46.1 & 214.4 \\
\hline $\mathrm{J}$ & 7.3 & 1797 & 1.58 & 1.05 & 1.55 & 314.3 & -10.3 & 46.0 & 37.8 & -70.0 & 332.8 \\
\hline $\mathrm{H}$ & 16.2 & 10425 & 1.55 & 0.88 & 1.55 & 706.6 & -93.9 & 245.5 & 128.9 & -171.8 & 786.1 \\
\hline K & 5.9 & 254 & 1.48 & 0.70 & 1.43 & 73.8 & -13.7 & 21.5 & 15.5 & -25.3 & 75.7 \\
\hline $\mathrm{F}$ & 4.9 & 105 & 1.09 & -2.72 & 0.91 & 24.1 & 1.9 & 10.9 & -11.2 & -8.8 & 53.0 \\
\hline $\mathrm{E}$ & 11.6 & 109 & 1.02 & -2.98 & 0.95 & 34.3 & 4.2 & 15.2 & -9.3 & -22.5 & 56.8 \\
\hline $\mathrm{C}$ & 7.5 & 100 & 1.01 & -1.88 & 1.14 & 28.0 & 4.1 & 6.2 & 2.3 & -5.1 & 69.4 \\
\hline D & 5.4 & 78 & 0.79 & -2.30 & 1.03 & 56.2 & 3.8 & 4.2 & -10.8 & -2.1 & 30.1 \\
\hline A & 4.3 & 203 & 1.02 & -2.00 & 1.55 & 30.5 & 9.1 & 8.7 & 6.0 & 5.7 & 228.6 \\
\hline B & 3.9 & 66 & 1.06 & -2.94 & 1.34 & 26.9 & 4.6 & 4.9 & 9.5 & -5.0 & 54.3 \\
\hline G & 5.6 & 133 & 1.49 & 2.09 & 1.05 & 163.9 & 0.6 & 3.8 & 2.0 & -3.7 & 32.3 \\
\hline $\mathrm{L}$ & 5.3 & 40 & 1.40 & 0.75 & 0.84 & 43.7 & 2.5 & 1.4 & -7.0 & -2.9 & 34.5 \\
\hline M & 3.7 & 144 & 1.47 & 1.64 & 1.44 & 72.9 & 2.1 & 4.8 & 1.9 & -7.9 & 72.9 \\
\hline $\mathrm{N}$ & 3.1 & 74 & 1.12 & 0.14 & 1.49 & 25.3 & 3.2 & 3.1 & 9.9 & 0.9 & 83.0 \\
\hline $\mathrm{O}$ & 2.1 & 247 & 1.54 & -1.89 & 1.48 & 170.8 & -0.7 & 3.7 & -4.1 & 3.1 & 98.7 \\
\hline $\mathrm{P}$ & 3.2 & 206 & 1.24 & -2.98 & 1.49 & 48.0 & 8.2 & 7.3 & -4.9 & -6.6 & 155.6 \\
\hline Q & 1.7 & 25 & 0.86 & -0.37 & 1.01 & 28.4 & 1.5 & 1.2 & 3.4 & 0.1 & 19.5 \\
\hline
\end{tabular}


To use our structural codes directly for the structural comparison, a score matrix similar to the BLOSUM for amino acids is desired. Using the alignments for representative structures in the database FSSP, which contains 2,860 sequence families representing 27,181 protein structures, we have constructed a substitution matrix called CLESUM for the conformational letters. The structures of the representative set are converted to their structural code sequences. All the pair alignments of the FSSP for the proteins with a sufficient similarity in the representative set are collected for counting aligned pairs of conformational letters. The total number of letter pairs is $1,284,750$. An entry of the matrix is the log-ratio of the observed frequency of the aligned corresponding pair to the expected frequency from a random alignment simply by chance. The substitution matrix is derived in the same way as BLOSUM was obtained (without clustering). The matrix is shown in Table 2, where a scaling factor of 20 instead of 2 is used to show more details (corresponding to 0.05 bit units).

To the best of our knowledge, CLESUM is the first substitution matrix directly derived from structure alignments for a conformational alphabet. (Matsuda et al. [13] introduced a backbone encoding which is a uniform partition of the 2D phase space of one bending and one torsion angles by mapping the phase space into the surfaces of a icosahedron; their substitution matrix is given by the cosine of the angles between the corresponding normals of the icosahedron. This matrix measures a geometrical similarity while CLESUM reflects an 'evolutionary' similarity.)

Table 2. CLESUM: The conformation letter substitution matrix (in 0.05 bit units).

\begin{tabular}{|c|c|c|c|c|c|c|c|c|c|c|c|c|c|c|c|c|c|}
\hline $\mathrm{J}$ & 37 & & & & & & & & & & & & & & & & \\
\hline $\mathrm{H}$ & 13 & 23 & & & & & & & & & & & & & & & \\
\hline I & 16 & 18 & 23 & & & & & & & & & & & & & & \\
\hline $\mathrm{K}$ & 13 & 5 & 21 & 49 & & & & & & & & & & & & & \\
\hline $\mathrm{N}$ & -2 & -34 & -11 & 28 & 90 & & & & & & & & & & & & \\
\hline$Q$ & -44 & -87 & -62 & -24 & 32 & 90 & & & & & & & & & & & \\
\hline $\mathrm{L}$ & -32 & -62 & -41 & -1 & 8 & 26 & 74 & & & & & & & & & & \\
\hline G & -21 & -51 & -34 & -13 & -8 & 8 & 29 & 69 & & & & & & & & & \\
\hline M & 16 & -4 & 1 & 12 & 7 & -7 & 5 & 21 & 61 & & & & & & & & \\
\hline B & -57 & -96 & -74 & -50 & -11 & 12 & -12 & 13 & -13 & 51 & & & & & & & \\
\hline $\mathrm{P}$ & -34 & -60 & -49 & -36 & -3 & 7 & -12 & 5 & 8 & 42 & 66 & & & & & & \\
\hline A & -23 & -45 & -31 & -19 & 10 & 16 & -11 & -6 & -2 & 20 & 35 & 73 & & & & & \\
\hline 0 & -24 & -55 & -34 & 5 & 15 & -13 & -4 & -1 & 5 & -12 & 4 & 25 & 104 & & & & \\
\hline C & -43 & -77 & -56 & -33 & -5 & 29 & 0 & -4 & -12 & 7 & 4 & 13 & 3 & 53 & & & \\
\hline $\mathrm{E}$ & -93 & -127 & -108 & -84 & -43 & -6 & -21 & -22 & -47 & 15 & -5 & -25 & -48 & 3 & 36 & & \\
\hline$F$ & -73 & -107 & -88 & -69 & -32 & 3 & -16 & -5 & -33 & 7 & 0 & -20 & -30 & 20 & 26 & 50 & \\
\hline D & -88 & -124 & -105 & -81 & -44 & 14 & -22 & -31 & -49 & 13 & -10 & -17 & -42 & 21 & 22 & 21 & 52 \\
\hline & $\mathrm{J}$ & $\mathrm{H}$ & I & $\mathrm{K}$ & $\mathrm{N}$ & $Q$ & $\mathrm{~L}$ & $\mathrm{G}$ & M & B & $P$ & $\mathrm{~A}$ & 0 & C & E & F & D \\
\hline
\end{tabular}

\subsection{Finding AFPs of high CLESUM similarity scores}

Suppose that the pair of structures to be aligned is $P$ and $P^{\prime}$ with $P$ being the shorter. The coordinates $\left\{\mathbf{r}_{i}\right\}$ and $\left\{\mathbf{r}_{j}^{\prime}\right\}$ of $C_{\alpha}$ atoms of the two proteins are converted to the sequences $S$ and $S^{\prime}$ of conformational letters, respectively. Since each letter corresponds to a quadrupeptide unit, the length of $S\left(S^{\prime}\right)$ is shorter than that of $P\left(P^{\prime}\right)$ by three. By convention, we assign the first letter to the third residue, the second to the fourth, and so on, until finally the last letter is assigned to the last residue but one.

Consider two fragments of the same length $l$, one of which starts at residue $i$ of $P$ and the other at $j$ of $P^{\prime}$. The local structural similarity of the fragment pair may be measured by

$$
\sigma=\sum_{k=0}^{l-1} M\left(s_{i+k}, s_{j+k}^{\prime}\right),
$$

where $M(a, b)$ is the $(a, b)$-entry of the CLESUM, and $s_{i}$ and $s_{j}^{\prime}$ are the conformational letters of corresponding residues. Here we have kept the same index for a residue and its conformational letter. Setting a 
threshold $T$, if the pair score $\sigma \geq T$, we call the pair an AFP, which defines a correspondence ( $l$ residue duads). Comparing each string of $S$ of length $l$ with those of $S^{\prime}$, we find all the AFPs. When an AFP contains residues of SSEs at its ends, shifts of the AFP often also have a high similarity score, hence they also form AFPs. To remove such redundancy, we keep only the one with the highest score among the nearby AFPs which are shifts of each other and which share common duads. A width $w$ is set to restrict the maximum overlap for this shaving. After shaving we have a reduced list of the representative AFPs. We sort the list in descending order of scores. Usually, a small $l$ and a low $T$ will result in a long list of AFPs.

For a given long enough AFP, we can find a rigid transformation to superpose its two members and make the spatial deviation of its duad $C_{\alpha}$ atoms very small. Since an AFP is determined only by local similarity, a superposition valid for one AFP need not be valid for another. We define the spatial distance or separation between two members of a certain AFP under a given transformation by

$$
\delta=\max _{\left(\mathbf{r}_{i}, \mathbf{r}_{i^{\prime}}^{\prime}\right) \in \operatorname{AFP}}\left\{\left|x_{i}-x_{i^{\prime}}^{\prime}\right|,\left|y_{i}-y_{i^{\prime}}^{\prime}\right|,\left|z_{i}-z_{i^{\prime}}^{\prime}\right|\right\},
$$

where $\left(\mathbf{r}_{i}, \mathbf{r}_{i^{\prime}}^{\prime}\right)$ is a duad of the AFP after transformation, and $(x, y, z)$ denotes the $3 \mathrm{D}$ coordinates of $\mathbf{r}$. A small separation $\delta$ implies a good superposition of the two AFP members.

\subsection{The greedy 'zoom-in' strategy}

There is no clearly defined unique way to evaluate the quality of protein structure alignments. We adopt the standard of ProSup: the goal is to maximize the number $N_{e}$ of structurally equivalent residues subject to a fixed Euclidean distance cutoff $d_{0}$ for judging correspondence between a residue pair and a minimal aligned segment size $\rho$.

To balance speed with accuracy, we generate two lists of AFPs, one for $l=20$ with threshold $T_{20}=350$, and the other for $l=8$ with $T_{8}=0$. Any two helices are locally similar. Length 20 will exclude many such purely local coincidence. Length 8 is necessary for including most significant aligned pieces. We denote them as List-20 and List-8, respectively. The two lists can be generated in a single run. We expect that a significant alignment should contain at least one AFP of length around 20. Initial primary correspondences will be taken from the top $K$, say top ten, AFPs of the sorted List-20. If the list size is less than 10, 'top ten' means all.

Once an AFP is chosen, the transformation optimal to the AFP may be used to superimpose the two proteins. The separation $\delta$ of any AFP under the transformation can then be calculated. Some AFPs are consistent with each other. That is, under the transformation optimal to one of the AFPs, which is referred to as the center AFP or simply the center, some other AFPs will have a small separation $\delta$. By thinking in terms of graph theory, AFPs are nodes, and the center is the center node. At a fixed threshold $d$ for $\delta$, if the separation of an AFP is smaller than $d$, an edge is linked between the node of that AFP and the center. The center and its linked neighbors form a star tree or star. We define the size of a star as the total number of its nodes. Taking each of the top $K$ AFPs as a center, we find its neighbor nodes in the top $J$ AFPs of the sorted List-20, where $J \geq K$. The stars really used by CLePAPS are subject to a further restriction: for a given center, we search the sorted List-20 successively from the top for neighbors of the center, and add a new neighbor AFP only when it does not overlap with any existing neighbor AFPs. In this way, we obtain $K$ restricted stars. We sort them first by their sizes, and then by the similarity score $\sigma$ of their centers in descending order. We remove the stars whose centers are a neighbor of the first star. Then, we examine the next star, and remove the stars associated with its neighbors, and so on, until all stars are examined. Only the centers of the retained stars will be taken as an initial alignment seed. The effect of this star removal is twofold: removing seed redundancy and selecting the seeds which better reflect the global consistency.

The extension of an initial seed alignment is mainly done by blank-filling of the AFPs from List- 8 which are consistent with the seed. Blanks are residue positions not included in an existing correspondence. The transformation optimal to the seed AFP need not be globally optimal. We use a multi-step 'zoom-in' strategy, starting with a low precision to avoid local trapping. We first use a large cutoff $d_{1}$, say $8 \AA$, as the consistency criterion. That is, we add only the AFPs with $\delta<d_{1}$ to the existing correspondence. The procedure of blank-filling is greedy. The AFPs with a higher $\sigma$ have a priority to be filled. We examine the top half of 
the AFPs in the sorted List- 8 one by one from the top. If none of the residues of an AFP is contained in the existing correspondence, we calculate its separation $\delta$. If $\delta<d_{1}$, we add the AFP to the correspondence. When blank-filling is fulfilled, the transformation optimal to the enlarged correspondence is determined to update the superposition of the two proteins. In the next run of blank-filling, cutoff $d_{1}$ is reduced to $d_{2}$, and five-sixths of the AFPs in List-8 are examined. In a third run, $d_{2}$ is further reduced to $d_{3}$, and the whole list is examined. Usually, three runs of iteration are enough for obtaining a full alignment.

There are mainly two ways to update the correspondence. One is to keep the existing duads and add new ones. The other is to re-start with an empty correspondence and then fill in blanks with AFPs from List-8. The latter strategy is used in CLePAPS. In the final polishing stage, the AFPs which have only a limited overlap with the existing correspondence can also be used for blank-filling.

We speed up computation by means of marking. At the beginning, all AFPs in List- 8 are identified as 'unmarked'. If an AFP has no two contiguous residue pairs whose coordinate differences are both smaller than $d_{i}$, it will be marked as 'invalid', and then will never be examined again.

\subsection{Refinement by elongation and shrinking}

After blank-filling we obtain an alignment usually as disjunct pieces. Due to the finite size of AFPs and the redundancy removal by shaving, it is possible that an aligned piece can be elongated near its ends. Assume that the residue duad $\left(i, i^{\prime}\right)$ is at one end of an AFP. Thus, either $\left(i+1, i^{\prime}+1\right)$ or $\left(i-1, i^{\prime}-1\right)$ will be an outer residue pair. If the Euclidean distance of the pair is smaller than $d_{0}$ we elongate the aligned piece by joining the residue duad to it. More nearby residue pairs can be further examined for elongation. On the other hand, depending on the relative quantities of $d_{0}$ and $d_{3}$, the Euclidean distance between some residue duads on the aligned pieces may be greater than $d_{0}$. We remove such pairs from the alignment (as a shrinkage of the AFPs). Although elongation and shrinking may be conducted subsequently for every complete run of blank-filling, CLePAPS conducts elongation and shrinking only after the final run.

A finetuning version of refinement is as follows. In the final run of blank-filling, position confliction in protein $P^{\prime}$ is ignored so one residue of protein $P$ may correspond to several residues of protein $P^{\prime}$. We sort in ascending order of the root mean square deviations (RMSDs) all the AFPs which are candidates for filling, then add these AFPs into an empty correspondence list one at a time and avoid any position confliction. We perform possible elongation of each filled AFP to enlarge the correspondence. For any unfilled gap in protein $P$, we search List-8 from the top. If an AFP which covers the gap is found, we calculate the Euclidian distance of residue pairs whose positions coincide with the gap. When the distance of a pair is smaller than $d_{0}$, we check also the distances of nearby pairs by shifting protein $P^{\prime}$, and add the pair with the smallest distance to the correspondence.

A filter for a minimal aligned segment length $\rho$ may be finally applied. A further iteration of transformation would additionally improve the quality of the alignment. Once a global alignment is accomplished, the total number $N_{e}$ of equivalent residue pairs and the RMSD of the alignment are calculated as quality indicators. Despite the star removal two alignments generated from two star centers may still be very similar. We compare entries of the rotation matrices. If the greatest difference between two corresponding matrix elements (or relative difference) is above $\epsilon$, say 0.1, the two alignments are regarded as identical. A more careful criterion is the number of coincident residue duads, which is used by ProSup.

Structure comparison often yields several distinct alignments as multiple solutions. The existence of alternative alignments is mainly due to structure repeats at different levels ranging from secondary structure, supersecondary structure to domains.[10] Another source is the domain move. CLePAPS often reports several alignments and ranks them according to their $N_{e}$.

\section{Results}

\subsection{Finding AFPs in the test case: phycocyanin versus colicin A}

This test case was thoroughly studied by $\mathrm{CE}$ for tuning its operation parameters. The two proteins have PDB codes 1colA and 1cpcL. Here letters A and L are chain identifiers. The former is classified as a 'membrane 
and cell surface protein', and the latter as an 'all alpha protein' by SCOP.[18] Their lengths are 204 and 172, respectively. Only 197 residues of 1 colA are given with their coordinates.

A great proportion of both the protein structures is helices. It is expected that the number of AFPs should be large. Numbers of AFPs found at two different lengths $l$ and different CLESUM score thresholds $T$ before and after shaving are shown in Table 3 . The maximum overlap for shaving is set to be $i \times l / 2$, and three overlap ranges for shaving are examined. From the table it is clearly seen that the number of AFPs drops significantly for long $l$ and high $T$, and shaving dramatically reduces the number of AFPs. Finally, in CLePAPS the maximum overlap is set to be 20 for List-20 and 4 for List- 8 .

Table 3. Numbers of AFPs found at different lengths $l$ and CLESUM score thresholds $T$ before and after shaving. $N_{i}$ denotes the number of AFPs after shaving at overlap range $i \times l / 2$.

\begin{tabular}{rrrrrr}
\hline & & before & \multicolumn{3}{c}{ after shaving } \\
$l$ & $T$ & $N_{0}$ & $N_{1}$ & $N_{2}$ & $N_{3}$ \\
\hline \multirow{4}{*}{20} & 500 & 21 & 7 & 6 & 6 \\
& 400 & 164 & 45 & 42 & 41 \\
& 350 & 381 & 93 & 87 & 85 \\
& 300 & 717 & 156 & 140 & 135 \\
\hline \multirow{4}{*}{8} & 200 & 513 & 239 & 200 & 195 \\
& 150 & 4273 & 1512 & 1093 & 953 \\
& 100 & 7753 & 2462 & 1616 & 1245 \\
& 50 & 10350 & 3161 & 1960 & 1418 \\
& 0 & 13207 & 3942 & 2316 & 1547 \\
\hline
\end{tabular}

We have examined the pair 1colA: 1cpcL and many other pairs to optimize the operation parameters of CLePAPS. We use the default values of these parameters as shown in Table 4.

Table 4. Default parameters of CLePAPS.

\begin{tabular}{ccl}
\hline Symbol & Value & \multicolumn{1}{c}{ Meaning } \\
\hline$l_{l}$ & 20 & length of long AFPs \\
$T_{l}$ & 350 & similarity threshold for long AFPs \\
$K$ & 10 & number of long AFPs used as seed candidates \\
$J$ & 50 & number of long AFPs for building a star-tree \\
$l_{s}$ & 8 & length of short AFPs for blank-filling \\
$T_{s}$ & 0 & similarity threshold for short AFPs \\
$\rho$ & 4 & minimum length of aligned fragments \\
$d_{0}$ & $5 \AA$ & distance cutoff for evaluating overall alignment \\
$d$ & $10 \AA$ & separation threshold for star construction \\
$d_{1}$ & $8 \AA$ & separation cutoff for blank-filling in first run \\
$d_{2}$ & $6 \AA$ & separation cutoff for blank-filling in second run \\
$d_{3}$ & $5 \AA$ & separation cutoff for blank-filling in third run \\
$\epsilon$ & 0.1 & maximal difference for rotational matrix \\
& & entries of two 'identical' alignments \\
\hline
\end{tabular}

\subsection{The Fischer benchmark test}

A well-known comprehensive test set for assessing the performance of fold recognition methods is the benchmark of Fischer et al., which contains 68 pairs of proteins.[19] All pairs of the set are known to be structurally similar, but they have low sequence identity, ranging from $8 \%$ to $31 \%$ with an average of $18.6 \%$ and a standard deviation of 4.4. This set covers a wide range of protein families. We test the benchmark with CLePAPS. The results of the alignment are summarized in Table 5. Although AFPs reflect mainly the local similarity, the construction of star trees helps us to select seed AFPs for pivoting superposition. We rank seeds first according to the sizes of their center-stars, and then according to the similarity scores $\sigma$ of the center seeds. The last column of the table is the rank of the center seeds from which the optimal alignments are derived. 
Table 5. Test of CLePAPS on the Fischer benchmark. ID: protein PDB ID and an optional fifth letter for chain index; $L$ : protein length; Rank: the rank of the center star from whose center the optimal alignment is generated; $N_{e}$ : the number of aligned residues; rmsd: RMSD of the alignment. Nine pairs whose optimal alignments are not from the rank-1 seed AFPs are indicated with a superscript in the last column for ranks. Superscripts $a, b$ and $c$ correspond to three groups of the nine pairs, see text.

\begin{tabular}{|c|c|c|c|c|c|c|c|}
\hline \multicolumn{4}{|c|}{ Protein pair } & DALI & DALI-core & \multicolumn{2}{|c|}{ CLePAPS } \\
\hline ID & $L$ & ID & $L$ & $N_{e} / \mathrm{rmsd}$ & $N_{e} / \mathrm{rmsd}$ & $N_{e} / \mathrm{rmsd}$ & Rank \\
\hline $1 \mathrm{mdc}$ & 133 & 1ifc & 131 & - & - & $127 / 1.87$ & 1 \\
\hline 1npx & 447 & $3 \mathrm{grs}$ & 461 & $395 / 3.45$ & $347 / 2.40$ & $335 / 2.38$ & 1 \\
\hline 1onc & 104 & $7 \mathrm{rsa}$ & 124 & $97 / 1.86$ & $91 / 1.49$ & $91 / 1.56$ & 1 \\
\hline 1osa & 148 & $4 \mathrm{cpv}$ & 108 & $67 / 1.43$ & $67 / 1.43$ & $67 / 1.43$ & $2^{a}$ \\
\hline $1 \mathrm{pfc}$ & 111 & 3hlaB & 99 & $88 / 2.83$ & $74 / 2.14$ & $77 / 2.29$ & 1 \\
\hline $2 \mathrm{cmd}$ & 312 & $6 \mathrm{ldh}$ & 329 & $286 / 2.52$ & $269 / 2.05$ & $271 / 2.09$ & 1 \\
\hline 2pna & 104 & 1shaA & 103 & $92 / 2.62$ & $85 / 2.14$ & $85 / 2.20$ & 1 \\
\hline 1bbhA & 131 & 2cсyA & 127 & $125 / 2.02$ & $121 / 1.86$ & $122 / 1.81$ & 1 \\
\hline $1 \mathrm{c} 2 \mathrm{rA}$ & 116 & $1 \mathrm{ycc}$ & 108 & $96 / 1.62$ & $95 / 1.53$ & $95 / 1.49$ & 1 \\
\hline $1 \mathrm{chrA}$ & 370 & $2 \mathrm{mnr}$ & 357 & $347 / 1.88$ & $340 / 1.73$ & $340 / 1.73$ & 1 \\
\hline $1 \mathrm{dxtB}$ & 147 & $1 \mathrm{hbg}$ & 147 & $135 / 2.04$ & $128 / 1.74$ & $137 / 1.95$ & 1 \\
\hline 2fbjL & 213 & 8 fabB & 214 & $194 / 2.30$ & $186 / 1.91$ & $186 / 1.90$ & 1 \\
\hline 1gky & 186 & 3adk & 194 & $154 / 2.97$ & $129 / 2.43$ & $122 / 2.44$ & 1 \\
\hline 1hip & 85 & 2hipA & 71 & $67 / 1.81$ & $66 / 1.71$ & $62 / 1.43$ & 1 \\
\hline 2sas & 185 & $2 \operatorname{scpA}$ & 174 & $168 / 3.58$ & $131 / 2.53$ & $134 / 2.49$ & 1 \\
\hline $1 \mathrm{fc} 1 \mathrm{~A}$ & 206 & $2 \mathrm{fb} 4 \mathrm{H}$ & 229 & $175 / 8.28$ & $101 / 2.01$ & $117 / 2.23$ & 1 \\
\hline $2 \mathrm{hpdA}$ & 457 & $2 \mathrm{cpp}$ & 405 & $374 / 3.47$ & $319 / 2.73$ & $307 / 2.64$ & 1 \\
\hline 1aba & 87 & lego & 85 & $72 / 2.19$ & $68 / 1.73$ & $68 / 1.73$ & 1 \\
\hline 1eaf & 243 & 4cla & 213 & $174 / 2.62$ & $161 / 2.25$ & $165 / 2.35$ & 1 \\
\hline $2 \mathrm{sga}$ & 181 & $5 p t p$ & 222 & $147 / 2.71$ & $128 / 1.87$ & $139 / 2.02$ & 1 \\
\hline $2 \mathrm{hhmA}$ & 278 & 1fbpA & 316 & $224 / 2.85$ & $195 / 2.11$ & $203 / 2.27$ & 1 \\
\hline 1aaj & 105 & $1 \mathrm{paz}$ & 120 & $80 / 1.67$ & $79 / 1.56$ & $79 / 1.61$ & 1 \\
\hline $5 \mathrm{fd} 1$ & 106 & liqz & 81 & $57 / 2.62$ & $47 / 1.95$ & $51 / 1.53$ & $5^{a}$ \\
\hline 1isuA & 62 & 2hipA & 71 & $58 / 2.28$ & $51 / 1.55$ & $54 / 1.98$ & 1 \\
\hline 1gal & 581 & $3 \operatorname{cox}$ & 500 & $401 / 3.05$ & $338 / 2.19$ & $342 / 2.18$ & 1 \\
\hline 1cauB & 184 & 1cauA & 181 & $162 / 2.18$ & $153 / 1.80$ & $154 / 1.82$ & 1 \\
\hline $1 \mathrm{hom}$ & 68 & 1lfb & 77 & $56 / 1.95$ & $52 / 1.40$ & $55 / 1.65$ & 1 \\
\hline 1tlk & 103 & 2rhe & 114 & $89 / 2.02$ & $81 / 1.41$ & $86 / 1.70$ & 1 \\
\hline 2omf & 340 & 2 por & 301 & $261 / 2.68$ & $231 / 2.06$ & $228 / 2.06$ & 1 \\
\hline $1 \operatorname{lga} A$ & 343 & 2 сур & 293 & $261 / 2.44$ & $235 / 1.84$ & $241 / 1.92$ & 1 \\
\hline 1mioC & 525 & $2 \min B$ & 522 & $412 / 3.61$ & $353 / 2.59$ & $361 / 2.63$ & 1 \\
\hline
\end{tabular}

Amongst 68 protein pairs, the centers of $59(87 \%)$ rank-1 star trees of List-20 lead to optimal alignments. The nine protein pairs whose optimal alignments do not correspond to a rank- 1 star tree form three groups $a, b$ and $c$. Four pairs in group $a$ have structural repeats, and the rank- 1 stars do correspond to one of the multiple best choices although their $N_{e}$ are relatively lower. For the two pairs in group $b$, the alignments from the rank-1 stars are similar to the optimal. For one pair of the two, 1 dsbA: $2 \operatorname{trx}$ A, the rank-1 center AFP is consistent with the optimal alignment. However, it leads to only partial alignment due to local trapping. An extra 'zoom-in' step with cutoff $10 \AA$ is able to obtain the optimal alignment from that AFP. The other three of group $c$ (1aep: 256bA, 1rcb: 2gmfA and 1bgeB: 2gmfA) belong to a 'four-helical up-and-down bundle'. The alignments of DALI consist of almost just helical regions; DALI and CE have nothing in common for the alignment between 1aep and 256bA. It seems that the evidence of local similarity to support the global alignments of these three pairs is not as strong as for other pairs in the benchmark. We shall come back to the pair 1bgeB: $2 \mathrm{gmfA}$ in the next subsection. It seems that the loss of sensitivity by keeping only the 
rank-1 star and obtaining alignment only from its center is rather limited.

(Table 5, continued)

\begin{tabular}{|c|c|c|c|c|c|c|c|}
\hline \multirow[b]{2}{*}{ ID } & \multicolumn{3}{|c|}{ Protein pair } & \multirow{2}{*}{$\begin{array}{c}\text { DALI } \\
N_{e} / \mathrm{rmsd}\end{array}$} & \multirow{2}{*}{$\begin{array}{r}\text { DALI-core } \\
N_{e} / \mathrm{rmsd}\end{array}$} & \multicolumn{2}{|c|}{ CLePAPS } \\
\hline & $L$ & ID & $L$ & & & $N_{e} / \mathrm{rmsd}$ & Rank \\
\hline $4 \mathrm{sbvA}$ & 199 & 2 tbvA & 287 & $162 / 2.09$ & $154 / 1.47$ & $154 / 1.47$ & 1 \\
\hline $8 \mathrm{i} 1 \mathrm{~b}$ & 146 & $4 \mathrm{fgf}$ & 124 & $118 / 2.47$ & $110 / 1.96$ & $110 / 2.08$ & $2^{a}$ \\
\hline $1 \mathrm{hrhA}$ & 125 & $1 \mathrm{rnh}$ & 148 & $114 / 1.98$ & $105 / 1.40$ & $105 / 1.37$ & 1 \\
\hline 1mup & 157 & $1 \mathrm{rbp}$ & 174 & $140 / 2.92$ & $123 / 2.12$ & $120 / 2.10$ & 1 \\
\hline $1 \mathrm{cpcL}$ & 172 & $1 \mathrm{colA}$ & 197 & $114 / 3.61$ & $99 / 3.02$ & $104 / 2.98$ & 1 \\
\hline 2ak3A & 226 & $1 \mathrm{gky}$ & 186 & $149 / 3.00$ & $120 / 2.35$ & $118 / 2.41$ & 1 \\
\hline $1 \operatorname{atn} \mathrm{A}$ & 373 & 1atr & 383 & $292 / 3.00$ & $257 / 2.34$ & $258 / 2.32$ & 1 \\
\hline $1 \mathrm{arb}$ & 263 & $5 p t p$ & 222 & $189 / 2.89$ & $164 / 2.10$ & $159 / 2.13$ & 1 \\
\hline 2pia & 321 & $1 \mathrm{fnb}$ & 296 & $216 / 2.52$ & $196 / 1.98$ & $195 / 2.12$ & $4^{b}$ \\
\hline 3rubL & 441 & $6 x i a$ & 387 & $206 / 4.14$ & $142 / 3.30$ & $154 / 2.94$ & $2^{a}$ \\
\hline $2 \mathrm{sar} A$ & 96 & 9rnt & 104 & $71 / 3.18$ & $58 / 2.46$ & $61 / 2.66$ & 1 \\
\hline $3 \mathrm{~cd} 4$ & 178 & 2rhe & 114 & $94 / 2.60$ & $88 / 1.49$ & $89 / 1.63$ & 1 \\
\hline 1aep & 153 & $256 \mathrm{bA}$ & 106 & $74 / 1.78$ & $73 / 1.63$ & $84 / 2.83$ & $4^{c}$ \\
\hline $2 \mathrm{mnr}$ & 357 & $4 \mathrm{enl}$ & 436 & $285 / 3.43$ & $237 / 2.72$ & $227 / 2.71$ & 1 \\
\hline 1ltsD & 103 & 1bovA & 69 & $67 / 1.92$ & $66 / 1.82$ & $66 / 1.91$ & 1 \\
\hline $2 \mathrm{gbp}$ & 309 & 2liv & 344 & $260 / 6.75$ & $109 / 2.37$ & $140 / 2.51$ & 1 \\
\hline $1 \mathrm{bbt}$ & 186 & $2 \mathrm{plv}$ & 288 & $168 / 2.62$ & $159 / 2.31$ & $150 / 2.19$ & 1 \\
\hline $2 \mathrm{mtaC}$ & 147 & 1 ycc & 108 & $80 / 2.10$ & $79 / 1.99$ & $71 / 1.94$ & 1 \\
\hline 1 tahA & 318 & 1 tca & 317 & $188 / 2.47$ & $178 / 2.15$ & $173 / 2.22$ & 1 \\
\hline $1 \mathrm{rcb}$ & 129 & $2 \mathrm{gmfA}$ & 121 & $82 / 3.32$ & $67 / 2.32$ & $82 / 2.77$ & $2^{c}$ \\
\hline $1 \mathrm{sacA}$ & 204 & $2 a y h$ & 214 & $133 / 3.03$ & $118 / 2.67$ & $132 / 2.79$ & 1 \\
\hline $1 \mathrm{dsbA}$ & 188 & $2 \operatorname{trx} A$ & 109 & $82 / 2.77$ & $77 / 2.04$ & $77 / 2.01$ & $2^{b}$ \\
\hline 1 stfI & 98 & $1 \mathrm{molA}$ & 94 & $85 / 1.92$ & $85 / 1.92$ & $85 / 2.29$ & 1 \\
\hline 2afnA & 331 & 1aozA & 552 & $248 / 2.56$ & $231 / 2.23$ & $223 / 2.24$ & 1 \\
\hline 1fxiA & 96 & $1 \mathrm{ubq}$ & 76 & $60 / 2.58$ & $55 / 2.30$ & $50 / 2.30$ & 1 \\
\hline 1bgeB & 159 & $2 \mathrm{gmfA}$ & 121 & $94 / 3.33$ & $79 / 2.22$ & $82 / 2.41$ & $2^{c}$ \\
\hline 3hlaB & 99 & 2rhe & 114 & $75 / 3.03$ & $63 / 2.32$ & $65 / 2.30$ & 1 \\
\hline $3 \operatorname{chy}$ & 128 & 2 fox & 138 & $103 / 3.04$ & $91 / 2.59$ & $86 / 2.76$ & 1 \\
\hline 2azaA & 129 & $1 \mathrm{paz}$ & 120 & $81 / 2.26$ & $78 / 1.92$ & $78 / 2.30$ & 1 \\
\hline 1cew & 108 & $1 \mathrm{molA}$ & 94 & $81 / 2.46$ & $76 / 2.14$ & $78 / 1.98$ & 1 \\
\hline 1cid & 177 & 2rhe & 114 & $97 / 3.15$ & $82 / 2.03$ & $87 / 2.21$ & 1 \\
\hline $1 \mathrm{crl}$ & 534 & 1ede & 310 & $211 / 3.47$ & $168 / 2.49$ & $169 / 2.65$ & 1 \\
\hline $2 \operatorname{sim}$ & 381 & 1nsbA & 390 & $292 / 3.26$ & $240 / 2.50$ & $248 / 2.61$ & 1 \\
\hline 1ten & 89 & $3 \mathrm{hhrB}$ & 195 & $86 / 1.91$ & $84 / 1.73$ & $84 / 1.71$ & 1 \\
\hline 1tie & 166 & $4 \mathrm{fgf}$ & 124 & $114 / 3.06$ & $97 / 2.18$ & $100 / 2.26$ & 1 \\
\hline $2 \mathrm{snv}$ & 151 & 5 ptp & 222 & $131 / 3.09$ & $113 / 2.38$ & $118 / 2.49$ & 1 \\
\hline $1 \mathrm{gp} 1 \mathrm{~A}$ & 183 & $2 \operatorname{trx} A$ & 109 & $97 / 3.70$ & $74 / 3.40$ & $85 / 2.22$ & 1 \\
\hline
\end{tabular}

Indeed, with the aid of the star tree construction, the AFPs in terms of CLESUM scores are efficient for generating protein structure alignment. The selection of a seed for initial correspondence plays an essential role in the quality of the final alignment. In most cases (41 of 68) the AFP of List-20 with the highest $\sigma$ is the one from which the optimal alignment is derived. It appears that $K=10$ is enough to include the seed leading to the optimal alignment. A large value of $J$ for construction of a star tree is favorable for picking up a right seed. The size of a star tree is small for a small $J$. When the structures to be compared are large or contain repeats, usually a large $J$ is required for star tree construction. For example, the pair 2 sim: $1 \mathrm{nsbA}$ is a highly repeated fold '6-bladed beta-propeller' with lengths 381 and 390 , respectively. At $J=30$, every star tree is of size 1 , consisting of only the center itself. In CLePAPS we use $J=50$; increasing $J$ from 30 to 50 does not add much extra computational cost. 
In the table we have also listed $N_{e}$ and RMSD of DALI alignments. It is difficult to compare them directly with those from CLePAPS. To make a close comparison, we derive a 'DALI core' alignment from the original DALI alignment as follows. We superimpose a given pair structure according to the DALI alignment, and calculate the distance of each correspondent residue duad. We remove residue duads with distance greater than $d_{0}$, and the aligned segments whose lengths are smaller than $\rho$. The remaining reduced correspondence is the DALI core of the original alignment. The transformation optimal to the core is then determined, and $N_{e}$ and RMSD of the core are calculated; they are also listed in the table. It is seen that the performance of CLePAPS is comparable with DALI. A more detailed comparison on ten protein pairs is given in the next subsection.

\subsection{Ten 'difficult' protein structure pairs}

Ten protein pairs from the Fischer benchmark set of 68 pairs were regarded as 'difficult' for fold recognition, and treated as a test set by CE and ProSup. The comparison of CLePAPS with DALI, CE and ProSup is shown in Table 6. Since different criteria are used there is no simple direct comparison. For example, a high RMSD would lead to a large number of equivalent residues. With an extra restriction in the minimal size for aligned segments, ProSup usually has a smaller $N_{e}$ than others. To make a direct comparison, as done in the last subsection for DALI alignments, we derive also CE-core alignments from the original CE alignments. Generally, alignments of CLePAPS are comparable with those of other alignment tools.

The two proteins of pair 1fxiA: 1ubq are of lengths 96 and 76 , respectively. There is only one member in List-20. The alignment listed in Table 5 is from the only AFP. This is rather risky, being prone to local trapping. It is easy for CLePAPS to give a warning. A simple way of rescue is to use a weaker criterion for AFPs, e.g. List-12 with $T_{12}=180$ or a lower $T_{20}$. The alignment listed in Table 6 is obtained by using either $T_{20}=200$ or $T_{12}=180$. The CLePAPS alignment for the pair 1bgeB: 2gmfA has nothing in common with the first of DALI's three alignments. The List-20 of the protein pair has 31 members, but none coincides with any segment of the first DALI alignment. This means that the local similarity of the alignment is rather weak. The CLePAPS alignment for the pair is very similar to the second DALI alignment of $N_{e}=94$ with RMSD 3.3A. Of the total 82 aligned residue duads, 72 are identical with those of DALI.

Table 6. Comparison of structure alignments obtained by DALI, CE, ProSup and CLePAPS for '10 difficult' cases from the Fischer benchmark. $N_{e}$ : total number of equivalent residue duads; rmsd: RMSD in units of $\AA$; IDA: number of residue duads which are identical to those of DALI.

\begin{tabular}{llrrrrrrrr}
\hline & & CE & DALI & CE-core & DALI-core & \multicolumn{2}{c}{ ProSup } & \multicolumn{2}{c}{ CLePAPS } \\
\multicolumn{2}{c}{ Pair } & $N_{e} /$ rmsd & $N_{e} /$ rmsd & $N_{e} /$ rmsd & $N_{e} /$ rmsd & $N_{e} /$ rmsd & IDA & $N_{e} /$ rmsd & IDA \\
\hline 1fxiA & 1ubq & $64 / 2.8$ & $60 / 2.6$ & $59 / 2.5$ & $55 / 2.3$ & $54 / 2.6$ & 41 & $55 / 2.4$ & 42 \\
1ten & 3hhrB & $87 / 1.9$ & $86 / 1.9$ & $85 / 1.7$ & $84 / 1.7$ & $85 / 1.7$ & 79 & $84 / 1.7$ & 77 \\
3hlaB & 2rhe & $85 / 3.5$ & $75 / 3.0$ & $71 / 3.0$ & $63 / 2.3$ & $71 / 2.7$ & 37 & $65 / 2.3$ & 57 \\
2azaA & 1paz & $85 / 2.9$ & $81 / 2.5$ & $73 / 2.5$ & $76 / 2.1$ & $82 / 2.6$ & 8 & $78 / 2.3$ & 72 \\
1cewI & 1molA & $81 / 2.3$ & $81 / 2.3$ & $78 / 2.0$ & $78 / 1.9$ & $76 / 1.9$ & 68 & $78 / 2.0$ & 75 \\
1cid & 2rhe & $98 / 3.0$ & $97 / 3.2$ & $79 / 2.0$ & $82 / 2.0$ & $84 / 2.3$ & 70 & $87 / 2.2$ & 72 \\
1crl & 1ede & $220 / 3.9$ & $211 / 3.5$ & $155 / 2.5$ & $168 / 2.5$ & $161 / 2.6$ & 147 & $169 / 2.7$ & 146 \\
2sim & 1nsbA & $276 / 3.0$ & $292 / 3.3$ & $236 / 2.5$ & $240 / 2.5$ & $248 / 2.6$ & 231 & $248 / 2.6$ & 213 \\
1bgeB & 2gmfA & $109 / 4.6$ & $94 / 3.3$ & $62 / 2.7$ & $79 / 2.2$ & $87 / 2.4$ & 0 & $82 / 2.4$ & 0 \\
1tie & 4fgf & $117 / 3.0$ & $114 / 3.1$ & $99 / 2.3$ & $97 / 2.2$ & $101 / 2.4$ & 48 & $100 / 2.3$ & 94 \\
\hline
\end{tabular}

\subsection{Database search with CLePAPS}

Only 56 of the 68 protein structures in the Fischer benchmark are distinct. We take 4 proteins from the 68 probes of the benchmark as query structures: $2 \mathrm{mtaC}$ from Class $\alpha$ Fold Cytochrome, 1fxiA from $\alpha+\beta$ Ubiquitin-like, 1tie from $\beta$ Trefoil, and 3chy from $\alpha / \beta$ Open sheet. Each query structure is aligned with each of the 56 target structures of the benchmark. The first eight structures found by CLePAPS to be similar to these probes are shown in Table 7 . In the benchmark, the structure counterparts of $2 \mathrm{mtaC}, 1 \mathrm{fxiA}, 1$ tie 
and 3chy are 1ycc, 1ubq, 4fgf and 2fox, respectively. It is seen that, indeed, 1ycc, 1 ubq and 4 fgf have the highest $N_{e}$ among the similar structures found. However, 2fox appears rather behind. A close inspection reveals that the six structures with a higher $N_{e}$ than 2 fox are rather large, about three or five times longer than 3chy (except 3adk), and all have a domain or repeat of the same CATH [20] topology 'Rossmann fold' as 3chy. Taking the lengths of the aligned proteins into account, the DALI $Z$-score [21] assigns the second highest value to 2 fox. The DALI $Z$-scores for alignments to 3chy are also listed in the table. All the listed structures found for 3chy are coincident with 3chy, at least at the CATH topology level.

According to the Fischer benchmark, four probe sequences 1tlk, 3cd4, 3hlaB and 1cid are associated with the structure 2rhe. We have also taken 2rhe as a query to search the 68 probes for similar folds, and the results are also listed in Table 7. As we may expect, the structures of the above four probes do indeed appear to be highly ranked among those similar to 2 rhe. However, an additional one, $2 \mathrm{fbjL}$, has the highest value of $N_{e}$. It is verified that the two domains of $2 \mathrm{fbjL}$ share the same SCOP superfamily or CATH homology with 2 rhe, and $2 \mathrm{fbjL}$ has the highest DALI $Z$-score. Protein 1 sacA is not classified as Immunoglobulin (IG) or IG-like, but it still shares the same CATH Architecture with 2rhe.

Table 7. Structures found by CLePAPS as highly similar to five queries. $Z_{\text {dali }}$ : the $Z$-score given by DALI; rmsd: RMSD in Âunits.

\begin{tabular}{|c|c|c|c|c|c|c|c|c|c|c|}
\hline \multicolumn{11}{|c|}{ Query structure } \\
\hline \multicolumn{2}{|c|}{$2 \mathrm{mtaC}$} & \multicolumn{2}{|c|}{ 1fxiA } & \multicolumn{2}{|c|}{1 tie } & \multicolumn{3}{|c|}{ 3chy } & \multicolumn{2}{|c|}{ 2rhe } \\
\hline ID & $N_{e} / \mathrm{rmsd}$ & ID & $N_{e} / \mathrm{rmsd}$ & ID & $N_{e} / \mathrm{rmsd}$ & ID & $N_{e} / \mathrm{rmsd}$ & $Z_{\text {dali }}$ & ID & $N_{e} / \mathrm{rmsd}$ \\
\hline $1 \mathrm{ycc}$ & $71 / 1.9$ & $1 \mathrm{ubq}$ & $50 / 2.3$ & 4 fgf & $100 / 2.3$ & 2 2liv & $102 / 2.6$ & 7.9 & $2 \mathrm{fbjL}$ & $102 / 1.5$ \\
\hline 1 tca & $47 / 3.1$ & $1 \mathrm{rbp}$ & $48 / 3.0$ & $5 p t p$ & $60 / 2.9$ & $2 \mathrm{minB}$ & $97 / 2.9$ & 6.7 & $3 \mathrm{~cd} 4$ & $89 / 1.6$ \\
\hline $1 \mathrm{hbg}$ & $47 / 2.6$ & $5 p t p$ & $44 / 3.2$ & 9rnt & $51 / 2.5$ & 1 tca & $96 / 2.6$ & 5.6 & 1cid & $87 / 2.2$ \\
\hline 1atr & $46 / 3.0$ & 1aozA & $39 / 3.1$ & 1cauA & $49 / 2.7$ & 1ede & $88 / 2.7$ & 4.9 & 1tlk & $86 / 1.7$ \\
\hline $2 \mathrm{scpA}$ & $44 / 2.9$ & $1 \mathrm{nsbA}$ & $38 / 3.4$ & 2plv1 & $49 / 2.8$ & $3 \mathrm{adk}$ & $88 / 3.0$ & 4.3 & $1 \mathrm{fc} 1 \mathrm{~A}$ & $76 / 2.6$ \\
\hline $2 \mathrm{cpp}$ & $43 / 2.9$ & 1atr & $35 / 2.7$ & $1 \mathrm{rbp}$ & $45 / 2.5$ & 6ldh & $87 / 2.7$ & 6.0 & 1ten & $68 / 2.8$ \\
\hline $4 \mathrm{cpv}$ & $42 / 3.2$ & 1ifc & $35 / 2.5$ & $1 \mathrm{fnb}$ & $45 / 2.9$ & 2 fox & $86 / 2.8$ & 6.8 & 1sacA & $67 / 2.6$ \\
\hline $2 \mathrm{mnr}$ & $41 / 2.8$ & $1 \mathrm{fbpA}$ & $35 / 2.8$ & 1nsbA & $45 / 3.4$ & $1 \mathrm{fnb}$ & $84 / 2.9$ & 3.1 & $3 \mathrm{hlaB}$ & $65 / 2.3$ \\
\hline
\end{tabular}

\subsection{Multiple solutions of alignments}

To be less greedy, CLePAPS generates several alignments from star centers of highly scored AFPs. Often there is one alignment which has a much higher $N_{e}$ than others. (A better measure of significance for alignments is the $P$-value or $Z$-score.) There are situations where several meaningful alignments do exist. We see such examples in the Fischer benchmark.

Proteins 8i1b and 4 fgf belong to SCOP fold beta-Trefoil, which exhibits a three fold rotational symmetry. Indeed, the alignment ranks 1 to 3 clearly demonstrate this symmetry. A more complicated example is the pair 2sim: 1nsbA. Both belong to SCOP fold 6-bladed beta-propeller, but in PFAM [22] classification 2sim has four repeats of PF02012 while 1nsbA is PF00064. Multiple alignments reflect the rough symmetry. Another example is the pair 3rubL: 6xia, which contains a SCOP fold TIM beta/alpha-barrel. Among the multiple alignments, only one follows a sequential order, while all the others correspond to a circular permutation. There are, however, non-topological alignments not related to a rotational symmetry, which will be described in the next subsection.

Proteins 1osa and 4cpv belong to all alpha SCOP fold EF hand-like. The former contains two EF hand domains, while the latter has a single domain. The top two alignments are mainly the two ways of alignments of the EF hand domains. Another similar example is the pair 3hhrB: 1ten of SCOP fold IG-like beta-sandwich.

Proteins 2gbp and 2liv both belong to SCOP class $\alpha / \beta$ superfamily Periplasmic binding protein-like I, consisting of two CATH 3-Layer $(\alpha \beta \alpha)$ Sandwich domains, which will be denoted by I and II with I near the N-terminus. The two domains present a repeat of highly similar $\alpha \beta \alpha$ segments. For example, the AFP search at length 20 of $2 \mathrm{gbp}$ against itself discovers a region of contiguous AFPs with the correspondence of sites $23-60$ to $163-200$ around an AFP of $\sigma=772$. The ranks 1 to 3 in CLePAPS alignments of $2 \mathrm{gbp}$ to 
2 liv are mainly the alignments of domain I to I, II to I, and II to II, respectively. The AFP search of $2 \mathrm{gbp}$ against 2liv ascertains a long piece of contiguous AFPs with a correspondence of sites $24-80$ of $2 \mathrm{gbp}$ to $34-90$ of 2 liv around the AFP-20 of the highest $\sigma=804$, a piece with a correspondence of $167-201$ to $37-71$ around an AFP of $\sigma=733$, and another with a correspondence of $137-181$ to $133-177$ around an AFP of $\sigma=606$. These roughly correspond to the cores of the three alignments, which are of $N_{e}=141$ with RMSD $=2.54 \AA, N_{e}=137$ with $\mathrm{RMSD}=2.66 \AA$, and $N_{e}=112$ with RMSD $=2.40 \AA$, respectively. A relative move between the two domains with respect to the two proteins makes the RMSD for the alignment of the proteins as a whole rather high. This is seen in the DALI and CE alignments, which are of $N_{e}=260$ with RMSD $6.8 \AA$ and $N_{e}=252$ with RMSD $4.6 \AA$, respectively.

A global alignment involves the spatial arrangement of fragments. A domain move can destroy a global alignment as a rigid superposition. However, the alignment of the corresponding sequences of conformational codes is not affected by the domain move, so it is convenient for discovering conservative substructures of domains. A domain move appears in the structure evolution. It also occurs as conformational change of some flexible proteins in function. For example, an adenylate kinase (AKE) has a stable inactive conformation, in addition to an active form, i.e., the open and closed forms. They are represented by structures of PDBIDs 4akeA and 1akeA, respectively. Of course, they have the identical amino acid sequence. Their code sequences are also highly similar; only three code pairs are of a negative score. Their (positions, codes and scores) are (K47, HM, -4), (I116, HM, -4) and (V121, NI, -11), respectively. If we cut the two structures arround K47, V121 and D159 into four pieces we can align each piece pair extremely well. The code comparison can easily discover two cutting points. In order for structure alignment tools based on a rigid superposition to recognize the four aligned pieces a stringent criterion for deviation should be imposed for finding multiple solutions.

\subsection{Non-topological alignment and domain shuffling}

It is well known that the 3D structures of two proteins may be surprisingly similar in secondary structure element packing while the sequential order of their SSEs is completely different. We do not see any clear example of such non-topological alignment other than a simple rotation in the Fischer benchmark. A good example of such protein pairs is SCOP fold SH3-like barrel 1ihwA: 1sso (of lengths 52 and 62, respectively).[23] We represent an aligned fragment by the triplet $\left(a: a^{\prime}, w\right)$, where $w$ is the fragment length, and $a$ and $a^{\prime}$ are starting positions in the two proteins. The alignment of 1ihwA: 1sso consists of 5 pieces:

$$
(2: 26 ; 10),(13: 36 ; 10),(28: 0 ; 7),(36: 8 ; 11),(47: 20 ; 5) \text {. }
$$

and has $N_{e}=43$ with RMSD 2.21A. The first two segments show that the N-terminus of 1ihwA aligns with the C-terminus of 1sso while the other three segments are the alignment between the C-terminus of 1ihwA and the N-terminus of 1sso. Such alignments cannot be found by algorithms using dynamic programming.

\section{Discussion}

CLePAPS distinguishes itself from other existing algorithms for pairwise structure alignment in its use of conformational letters. The description of 3D segmental structural states by a few conformational letters aptly balances precision with simplicity. The substitution matrix CLESUM provides us with a proper measure of the similarity between these discrete states or letters. Such a description fits the $\epsilon$-congruent problem very well. Furthermore, CLESUM extracted from the database FSSP of structure alignments contains information of structure database statistics. For example, although two frequent helical states are geometrically very similar, scores between them are relatively low, which reduces the chance of accidental matching of two irrelevant helices. The conversion of coordinates of a 3D structure to its conformational codes requires little computation. Once we transform 3D structures to $1 \mathrm{D}$ sequences of letters, tools for analyzing ordinary sequences can be applied with some modification. The use of conformational letters for fast local similarity search can be integrated into many existing tools to improve the latter's efficiency. 
Recently, a few research groups have developed substitution matrices using various coding schemes for structural alphabets. For example, the coding of Godzik's group is based on a rigid fragment library, and their matrices were constructed using multiple structure alignments (HOMSTRAD) and alignments derived from multiple sequence alignments (BLOCKS).[24] Tyagi et al. published a matrix for their alphabet of 16 'protein blocks' representing eight dihedral angles of pentapeptides.[25] Also, Chang et al. reported a coding based on writhing number defined for a chain fragment and a matrix generated by using the CLASTALW alignments of sequences derived from SCOP as lineages.[26] Tung et al. derived a structural alphabet by clustering in a plot of two angles of pentapeptides, and its substitution matrix SASM.[27] Lo et al. derived another substitution matrix for their Ramachandran codes.[28] Structural codes convert structures into one-dimensional sequences. These authors then compared structures by methods of sequence alignment or database search, but no structure alignment was considered.

The CLESUM similarity score can be used to sort the importance of AFPs for a greedy algorithm, such as CLePAPS. Guided by CLESUM scores, only the top few structurally similar fragments need to be examined to determine the superposition for alignment, and hence a reliable greedy strategy becomes possible. Since many computational steps are conducted on conformational codes instead of $3 \mathrm{D}$ coordinates it runs faster than other tools, especially for large proteins. The running time for the 68 pairs of the Fischer benchmark is less than 2 percent of that of the downloaded CE local version.

To a certain extent CLePAPS resembles some other algorithms such as STRUCTAL [29] and ProSup, but there are fundamental differences. Apart from its key feature in its use of the representation of conformational letters, CLePAPS is different from most algorithms in that it never conducts any dynamic programming, so it is able to obtain non-topological as well as topological alignments. However, the conformational alphabet can be used to find an initial correspondence for dynamic programming. We have tested two ways: the usual Needleman-Wunsch global alignment method [30] with some simple penalty for the gaps or a little more sophisticated way by joining nonoverlapping AFPs, which is the Needleman-Wunsch alignment of AFPs. A gap penalty is necessary to keep the aligned pieces less scattered (sequentially).

It should be remarked that suitable tuning of the parameters used by CLePAPS is somewhat crucial to its optimal performance. A large value of basis width $l$ or similarity threshold $T$ would reduce search times, but at the price of sensitivity. Our strategy is to use stringent parameters first for finding reliable seed matches by star tree construction to initiate the alignment, and then to fill missing blanks for eventually compensating the sensitivity loss with relaxed parameters. The 'zoom-in' strategy for blank-filling starts with a vague alignment to avoid local trapping, and then refines it in later steps. For two remote structures, to keep the size of List-20 reasonably large a lower $T_{20}$ is preferable. This also happens when one or both proteins are small. For large proteins a large $J$ is often necessary for obtaining a star large enough to avoid local trapping. However, CLePAPS gets warned by a too small degree of a star center, so the danger of local trapping can be significantly diminished. We have tested various combination of parameters. In general, CLePAPS is not extremely sensitive to the choice of parameters. For example, $T_{20}=300$ or an even lower value may be used in most cases to guarantee that the size of List-20 is large enough, and $T_{8}=50$ is often sensitive enough to include all relevant aligned segments.

The problem of evaluating the significance of a structural alignment by a $P$-value or $Z$-score is by no means simple. It has been demonstrated by Lackner et al. that optimizing the number of equivalent residues under a distance cutoff for residue equivalence provides a simple and intuitive measure of structure similarity. [10] The similarity index $\mathrm{SI}=\mathrm{RMSD} \times L_{\min } / N_{e}$ of Kleywegt and Jones is simple and convenient, where $L_{\min }$ is the shorter length of the two proteins.[31] The $Z$-score of DALI (with possible modification in $M(L)$ ) can be used as another practicable measure of significance. A detailed discussion of the $P$-value and $Z$-score is beyond the scope of this paper.

CLESUM counts only information of conformation. However, the FSSP alignments from which CLESUM was derived contain also amino acid information. The use of a modified CLESUM including also such information would elucidate biochemical roles in alignment.[16] The idea of using structural alphabets for pairwise structure alignment is also valid for multiple structure alignment, where an AFP is replaced by a similar fragment block. An algorithm has been developed along this line.[32]

This work is supported by the National Natural Science Foundation of China and National 
Basic Research Program of China (2007CB814800).

\section{References}

[1] Holm,L. and Sander,C. The FSSP database of structurally aligned protein fold families. Nucleic Acid Res., 1994; 22: 3600-3609.

[2] Holm,L. and Sander,C. Dali/FSSP classification of three-dimensional protein folds. Nucleic Acid Res., 1997; 25: 231-234.

[3] Madej,T., Gibrat,J.F., and Bryant,S.H. Threading a database of protein cores. Proteins: Struct. Funct. Genet. 1995; 23: 356-369.

[4] Gibrat,J.F., Madej,T., and Bryant,S.H. Surprising similarities in structure comparison. Current Opinion in Structural Biology 1996; 6: 377-385.

[5] Shindyalov,I.N., Bourne,P.E. Protein structure alignment by incremental combinatorial extension (CE) of the optimal path. Protein Engineering 1998; 11: 739-747.

[6] I Eidhammer, I Jonassen, WR Taylor, Structure comparison and structure patterns, J. Comput. Biol. $2000 ; 7: 685-716$.

[7] Koehl,P., 2006, Protein Structure Classification, Chapter 1 of Reviews in Computational Chemistry, V. 22, ed. KB. Lipkowitz, TR. Cundari, and VJ. Gillet, Wiley-VCH, John Wiley \& Sons, Inc., 2006.

[8] Kabsch,W. A discussion of the solution for the best rotation to related two sets of vectors. Acta. Crystal., 1978; 34A: 827-828.

[9] Umeyama,S. Least-Squares Estimation of Transformation Parameters between 2-Point Patterns, IEEE Trans. Pattern Anal. \& Machine Intel., 1991; 13:376-380.

[10] Lackner,P., Koppensteiner,W.A., Sippl,M.J., and Domingues,F.S. ProSup: a refined tool for protein structure alignment, Protein Engineering, 2000; 13: 745-752.

[11] Rooman,M.J., Kocher,J.-P.A., and Wodak,S.J. Prediction of protein backbone conformation based on seven structure assignments: Influnce of local interactions J. Mol. Biol., 1991; 221: 961-979.

[12] Park,B.H., and Levitt,M. The complexity and accuracy of discrete state models of protein structure. J. Mol. Biol., 1995; 249: 493-507.

[13] Matsuda H, Taniguchi F, Hashimoto A. An approach to detection of protein structural motifs using an encoding scheme of backbone conformations, Proc. Pac. Symp. Biocomput., 1997: 280-291.

[14] Edgoose,T., Allison,L. and Dowe,D.L. An MML classification of protein structure that knows about angles and sequences, Proc. 3rd Pacific Symposium on Biocomputing (PSB-98), Hawaii, USA, 1998: 585-596.

[15] Camproux,A.C., Tuffery,P., Chevrolat,J.P., Boisvieux,J.F., and Hazout,S. Hidden Markov model approach for identifying the modular framework of the protein backbone, Protein Eng., 1999; 12: 10631073.

[16] Zheng,W.M. and Liu,X. A protein structural alphabet and its substitution matrix CLESUM, Lecture notes in Bioinformatics 3680 (eds. C. Priami and A. Zelikovsky), Springer Verlag, Berlin, 2005: 59-67; http://arxiv.org/abs/q-bio/0412046.

[17] Holm,L., and Sander,C. Touring protein fold space with Dali/FSSP, Nucleic Acids Res., 1998; 26: 316-319.

[18] Murzin,A.G., Brenner,S.E. , Hubbard,T. , and Chothia,C. SCOP: A structural classification of proteins database for the investigation of sequences and structures. J. Mol. Biol., 1995; 247: 536-540.

[19] Fischer,D., Elofsson,A., Rice,D., and Eisenberg,D., Assessing the performance of fold recognition methods by means of a comprehensive benchmark, Proc. Pac. Symp. Biocomput., 1996: 300C318. 
[20] Orengo,C.A., Michie,A.D., Jones,S., Jones,D.T., Swindells,M.B., and Thornton,J.M. CATH - A hierarchic classification of protein domain structures, Structure, 1997; 5:1093-1108.

[21] Holm,L., and Sander,C. Dictionary of Recurrent Domains in Protein Structures, Proteins, 1998; 33: 88-96.

[22] Bateman,A., Coin,L., Durbin,R., et al. The Pfam protein families database, Nucleic Acids Res., 2004; 32:D138-D141.

[23] Jung,J. and Lee,B. Circularly permuted proteins in the protein structure database, Protein Sci., 2001; 10:1881-1886.

[24] Friedberg,I., Harder,T., Kolodny,R., Sitbon,E., Li,Z., and Godzik,A. Using an alignment of fragment strings for comparing protein structures. Bioinformatics. 2007; 23:e219-224.

[25] Tyagi,M., Gowri,V.S., Srinivasan,N., de Brevern,A.G., and Offmann,B., A substitution matrix for structural alphabet based on structural alignment of homologous proteins and its applications. Proteins 2006; 65: 32-39.

[26] Chang,P.L., Rinne,A.W., and Dewey,T.G., Structure alignment based on coding of local geometric measures. BMC Bioinformatics, 2006; 7:346.

[27] Tung,C.T., Huang,J.W., and Yang,J.M., Kappa-alpha plot derived structural alphabet and BLOSUMlike substitution matrix for rapid search of protein structure database, Genome Biology 2007; 8:R31.

[28] Lo,W.C., Huang,P.J., Chang,C.H., and Lyu,P.C., Protein structural similarity search by Ramachandran codes, BMC Bioinformatics 2007, 8:307.

[29] Subbiah,S., Laurents,D.V., and Levitt,M. Structural similarity of DNA-binding domains of bacteriophage repressors and the globin core. Curr. Biol., 1993; 3: 141C148.

[30] Needleman,S.B., and Wunsch,C.D. A general method aplicable to the search for similarity in the amino acid sequence of two proteins. J. Mol. Biol., 1970; 48: 443-454.

[31] Kleywegt,G.J., Jones,A. Superposition. CCP4/ESF-EACBM Newsletter on Protein Crystallog., 1994; 31:9C14.

[32] Liu,X., Zhao,Y., and Zheng,W.M. CLEMAPS: Multiple Alignment of Protein Structures, Proteins (to appear). 